\title{
Nigella sativa oil protected the hippocampus against Acetyl cholinesterase and oxidative dysfunctions-driven impaired working memory in rats
}

\author{
Imam Aminu ${ }^{1,2}$, Alakoso Jumoke Teslimat ${ }^{1}$, Williams Victoria ${ }^{2}$, Chengetanai \\ Samson ${ }^{2,3}$, Oyewole Lukuman Aboyeji ${ }^{4}$, Olaleye Olatunbosun ${ }^{5}$, Shittu Toyin Sheu- \\ Tijani $^{6}$, Ajao Moyosore Saliu ${ }^{1}$ \\ ${ }^{I}$ Neuroscience Unit, Department of Anatomy, College of Health Sciences, University of Ilorin, \\ Original \\ Nigeria. \\ Article \\ ${ }^{2}$ Comparative Neurobiology Unit, School of Anatomical sciences, Faculty of Health Sciences, \\ University of the Witwatersrand, Johannesburg, Republic of South Africa. \\ ${ }^{3}$ Division of Basic Medical Sciences, National University of Science and Technology, Zimbabwe. \\ ${ }^{4}$ Neurophysiology Unit, Department of Physiology, College of Health Sciences, University of \\ Ilorin, Nigeria. \\ ${ }^{5}$ Department of Oral science, Faculty of Health Sciences, University of the Witwatersrand, \\ Johannesburg, Republic of South Africa. \\ ${ }^{6}$ Endocrinology and metabolism Research Unit, Department of Physiology, College of \\ Medicine, University of Ibadan, Ibadan, Nigeria
}

\begin{abstract}
Oxidative damages in organophosphates poisoning is associated with neuro-cognitive deficits. This study investigates the protective effect of Nigella sativa oil (NSO) in mitigating dichlorvos (DDVP) induced oxidative damage and neurocognitive impairment in rats. Thirty-two rats were randomly divided into four groups, exposed to $1 \mathrm{ml} / \mathrm{kg}$ of normal saline, $8.8 \mathrm{mg} / \mathrm{kg}$ of DDVP, DDVP $+1 \mathrm{ml} / \mathrm{kg}$ of NSO and NSO respectively for 14 consecutive days. Body weights were recorded at day 1 and 15 of the experiment, the rats were exposed to 3 trials each on the 11, 12 and 13th days in the Morris water maze, and subsequently latency to hidden platform and time in the platform quadrant were recorded as measures of long term memory (LTM), short term memory (STM) and reference memory on the 14th day. The rats were euthanized on the 15th day, the brains excised and the hippocampi of five brains in each group were removed, homogenized to analyze for total reactive oxygen species (ROS), nitrous oxide (NO) levels and acetylcholinesterase (AChE) activities, while the other three were processed for histology and Ki67 immunohistochemistry. DDVP exposure caused a significant increase in hippocampal NO and ROS levels, with reductions in AChE activities and Ki67 protein expression. This was associated with delayed escape latency and reduced time in platform quadrant. NSO intervention prevented outburst in ROS and NO, preserved the neurogenic cells and improved neuro-cognitive indices. We thus conclude that stabilizing oxidative and neurogenic functions are vital to protect against DDVP hippocampal insults.
\end{abstract}

Received: 05 August 2018, Accepted: 14 November 2018

Key Words: Acetylcholinesterase; cognition; dichlorvos; nigella sativa oil; oxidative damage.

Corresponding Author: Imam Aminu, M.Sc., Neuroscience Unit, Department of Anatomy, Faculty of Basic Medical Sciences, University of Ilorin, P.M.B 1515, Ilorin 240003, Nigeria School of Anatomical Sciences, Faculty of Health Sciences, University of the Witwatersrand, 7 York Road, Parktown 2193, Johannesburg, Republic of South Africa, Tel.: +2348165663947, E-mail: imam.a@unilorin.edu.ng

Bulletin of Faculty of Pharmacy, Cairo University, ISSN: 1110-0931, Vol. 57, No. 1 


\section{INTRODUCTION}

Organophosphates (OPs) are used extensively worldwide and poisoning resulting from inadvertent exposure to humans is becoming endemic, especially in the third world nations, posing a serious public health concern with millions of reported cases annually. ${ }^{[1]}$ These toxicants are potent inhibitors of cholinesterases that lead to a massive build-up of presynaptic acetylcholine which induces an array of deleterious effects, including convulsions, oxidative damage and neurobehavioral deficits increasing the risk of a variety of chronic illnesses, including respiratory diseases, metabolic deregulations and neurologic disorders. ${ }^{[2,3]}$ The incidences of poisoning with OPs have doubled in the last decade, and are associated with poor quality of life, depression, anxiety and stress, ${ }^{[4]}$ impaired neuropsychological performances ${ }^{[5]}$ among others.

Dichlorvos (DDVP), one of the effective OPs used throughout the world, in household and agricultural activities for the control of insects and pests, whose primary mechanism of toxicity is an irreversible inhibition of acetylcholinesterase (AChE) activities, eliciting reproductive toxicity, ${ }^{[6]}$ hematotoxicity ${ }^{[7]}$ cardiotoxicity, ${ }^{[8]}$ and neurotoxicity ${ }^{[9]}$ in a host of other inevitable damages.

Memory, being a central factor to efficient functions is primarily encoded by the hippocampus ${ }^{[10]}$ a structure that is not only crucial to the acquisition of new memories, but intimately involved in cognitive pieces including learning and spatial cognition. ${ }^{[11]}$

Nigella sativa, commonly known as the black seed and belonging to the botanical family of Ranunculaceae, is extensively used in the treatment of different diseases. Nigella sativa oil (NSO) is a highly valued medicinal solvent, used traditionally in the treatment of many diseases. Evidence from animal and human studies has shown that the oil of Nigella sativa exhibits extensive pharmacological properties including antioxidant, ${ }^{[12,13]}$ anti-inflammatory, ${ }^{[14]}$ neuroprotective, ${ }^{[12,13,15]}$ immunomodulatory and antitumor, ${ }^{[7,16]}$ efficacy in neurodegenerative disease ${ }^{[17]}$ and memory enhancing effects. ${ }^{[18]}$ This study evaluated the neuroprotective efficacies of NSO's antioxidant and neurogenic potentials against DDVP induced hippocampal neurotoxicity.

\section{MATERIALS AND METHODS}

\subsection{Chemicals and Drugs}

Dichlorvos (PubChem substance ID 329756736) PESTANAL ${ }^{\circledR}$, analytical standard was purchased from Sigma (Sigma-Aldrich,St. Louis, MO, USA), while the normal saline solution was prepared in the laboratory. The Nigella sativa oil (concentration; $100 \%$ black seed; HUSNA black seed oil, Fazhab Agency, Karachi, Pakistan) was purchased from a TIBB-medical store in Ilorin, Kwara state, Nigeria.

\subsection{Animal Care}

Thirty-two adult male Wistar rats weighing between $150 \mathrm{~g}$ and $170 \mathrm{~g}$ were obtained from the University of Ilorin Biological garden, Ilorin. They were housed in cages and fed with standard rat chow and water ad libitum, in the animal holding unit of the Faculty of Basic Medical Sciences, College of Health Sciences, University of Ilorin, Ilorin. The rats were exposed to a 12 hours' light/dark cycle at room temperature for 7 days before the commencement of the experiments. All rats were handled in accordance with the standard guide for the care and use of laboratory animals.

\subsection{Treatment Schedule}

The rats were randomly divided into four groups $(n=8)$ as follows:

Group 1 (control)- were given normal saline $(1 \mathrm{ml} / \mathrm{kg}$ orally) daily for 14 days $^{[12,13]}$

Group 2- were given DDVP ( $8.8 \mathrm{mg} / \mathrm{kg}$ orally) daily for 14 days $^{[12,13]}$

Group 3- were given DDVP (8.8 mg/kg orally) plus NSO ( $1 \mathrm{ml} / \mathrm{kg}$ orally) daily for 14 days $^{[12,13,15,18]}$

Group 4- were given NSO ( $1 \mathrm{ml} / \mathrm{kg}$ orally) daily for 14 days. ${ }^{[12,13,15,18]}$

All procedures were scheduled and carried out during the early phase of the day between 07:00 and 08:30 am, and treatments were given for fourteen consecutive days.

\subsection{Ethical approval}

This research work was approved by the University of Ilorin Ethical Review committee, following the recommendation of the College of Health Sciences Ethical Review Committee, University of Ilorin, Ilorin, Nigeria. The research was approved to be in compliance with the Institutional Animal Care and Use Committee (IACUC).

\subsection{Body and Brain Weight measurements}

The body weights of all the rats were recorded after acclimatization on the first day of the exposures as initial weight and on the last day of exposure as the final weight. The differences between the two weights were calculated and recorded as the weight changes. The brain weights of all rats were recorded on day 15 after the sacrifice and a ratio of the brain to final body weight was calculated and recorded.

\subsection{Behavioral evaluations}

The rats were subjected to behavioral evaluations on the 14th day of the treatment to assess, Short term memory, Long term memory and Reference memory in the Morris Water Maze paradigm.

\subsubsection{Morris water maze procedure}

The Morris water maze (MWM) apparatus is the 
most commonly used model to test spatial learning and memory. To evaluate spatial memory, rats were tested in a circle shaped black pool filled with $23-24{ }^{\circ} \mathrm{C}$ water (pool dimensions: $60 \mathrm{~cm}$ deep $\times 136 \mathrm{~cm}$ diameter). The pool was divided to four quadrants with boundaries labelled north $(N)$, east (E), south (S) and west (W) and a circular platform (10 $\mathrm{cm}$ diameter, $28 \mathrm{~cm}$ high) was submerged about $2 \mathrm{~cm}$ below water surface in the central area of the southwest quadrant of the pool. Animals were allowed to swim until they found and remained on the platform for $15 \mathrm{~s}$. If they were not able to find the platform after $60 \mathrm{~s}$ of swimming, they were guided to the platform by examiner and were allowed to stay on it for $15 \mathrm{~s}$. The rats were then removed from the pool, dried and placed in their holding bin for 5 min. Trials were recorded by a video system. Animals received a training session consisting of three trials per session (once from each starting point) for 3 days (days 11, 12 and 13), with each trial having a maximum duration of $60 \mathrm{~s}$ and a trial interval of approximately $30 \mathrm{~s}$. Twenty-four hours after the acquisition phase, the time taken to locate the hidden platform (escape latency) was recorded as long term memory (LTM), and the average escape latency for trials 2 and 3 as the short term memory (STM). A probe test was conducted by removing the platform, and allowing the rats to swim freely in the pool for $60 \mathrm{~s}$; the time spent in the target quadrant which had previously contained the hidden platform was recorded as the reference memory ( $14^{\text {th }}$ day). The time spent in the target quadrant indicated the degree of relative memory consolidation which had taken place after learning. ${ }^{[18]}$

\subsection{Biochemical evaluation}

At the end of the treatment period, the animals were euthanized with an overdose of Ketamine $(10 \mathrm{mg} / \mathrm{kg}$ ip) and the brains were quickly dissected out and weighed. Hippocampal tissues (from Bregma $-2.5 \mathrm{~mm}$ to $-4.5 \mathrm{~mm}$ ) were removed from the brains of five rats from each group, dipped in $30 \%$ sucrose solution, homogenized and portions centrifuged at 2500 revolutions per minute for 10 minutes and the supernatant collected into tubes containing the compounds for the Reactive Oxygen Species (ROS) and Nitrous Oxide (NO) analysis. Nitric Oxide metabolites were measured using Griess reagent. Tissue samples were added to the Griess reagents, sulfanilamide and naphthyl ethylene diamine solutions. Absorbance was measured with the aid of a microplate reader and the levels of NO metabolites were calculated from standard curve. ${ }^{[19]}$

Since acetylcholinesterase inhibition is the pathological hallmark of organophosphate poisoning, AChE levels are frequently used for the diagnosis of organophosphate exposure. The remaining portions of the homogenized hippocampal tissues were placed in phosphate buffer with $1 \%$ Triton-X 100 and centrifuged at $5000 \mathrm{rpm}$ for 10 minutes. The following reagents were used; $35 \mu \mathrm{L}$ of $5 \mathrm{mM}$ dithio-bisnitrobenzoic acid (DTNB), also known as Ellman's reagent, $10 \mu \mathrm{L}$ of $75 \mathrm{mM}$ acetylthiocholine (ATCh) and $50 \mathrm{mM}$ phosphate buffer (pH 8.0). Protein concentration in brain homogenates was quantified using a Bradford assay and AChE activity was calculated in micromoles of ATCh hydrolysed per hour per milligram of protein.

\subsection{Tissue processing and Histopathology}

After euthanasia and extraction the brains of three rats from each group, the brains were fixed in $10 \%$ formalin for 24 hours, hippocampal blocks (from Bregma $-2.5 \mathrm{~mm}$ to $-4.5 \mathrm{~mm}$ ) were removed, dehydrated through ascending grades of alcohol, cleared in xylene and embedded in paraffin blocks. Thin hippocampal tissue sections $(5 \mu \mathrm{m}$ in thickness) were stained with Cresyl fast violet (CFV) for Nissl substances or immunostained to reveal Ki67 protein distributions.

\subsubsection{Immunohistochemistry for Ki-67}

The Ki-67 is a chromosome-associated protein present during division (G1, S, G2, and $M$ phases but absent from cells at rest, G0). Paraffin embedded sections were incubated for epitope retrieval in citrate buffer, $\mathrm{pH}$ 6.0, at 90 ${ }^{\circ} \mathrm{C}$ for 40 minutes, followed by incubation in endogenous peroxidase blocking reagent, $0.6 \% \mathrm{H} 2 \mathrm{O} 2$ in Tris-buffered saline (TBS)-Triton ( $0.05 \%$ Triton $\mathrm{X}-100$ in TBS, $\mathrm{pH} 7.4)$ for 30 minutes at room temperature. Thereafter, sections were pre-incubated in $2 \%$ serum (normal goat serum) + $0.1 \%$ bovine serum albumin (BSA) $+0.25 \%$ Triton in TBS for 60 minutes at room temperature. Afterwards, sections were incubated with polyclonal rabbit-anti-lyophilizedKi-67p antibody (Novocastra, Newcastle, UK; 1:5,000 in preincubation solution) overnight at $4{ }^{\circ} \mathrm{C}$. Incubation with biotinylated goat anti-rabbit IgG $(1: 1,000+2 \%$ normal goat serum $+0.1 \%$ BSA in TBS; Vector lab, CA, USA;1:250) was performed for 2 hours at room temperature followed by incubation with streptavidin-biotin complex (Vectastain Elite $\mathrm{ABC}$ kit) and stained with 3,3'-diaminobenzidine (DAB) as chromogen. Until incubation with primary antibody, all rinses in between incubations were made with TBS-Triton, afterwards with TBS alone.

\subsection{Statistical Analysis}

Data from the morphometry, behavior and biochemicals were analyzed using one-way analysis of variance (ANOVA) and subjected to post hoc Bonferroni's multiple comparison test. The results are expressed as mean \pm SEM. Statistical analyses were performed using Graphpad Prism software (version 5.0, La Jolla, CA). Values of $p \leq 0.05$ were considered statistically significant.

\section{RESULTS}

\subsection{NSO prevented DDVP induced body weight loss}

Administration of DDVP in rats produced a marked weight loss in the exposed rats. The co-administration of DDVP and NSO prevented weight loss in the treated rats, comparable to those observed in the control rats. However, NSO administration resulted in body weight gain when given on its own. (Fig. 1) 


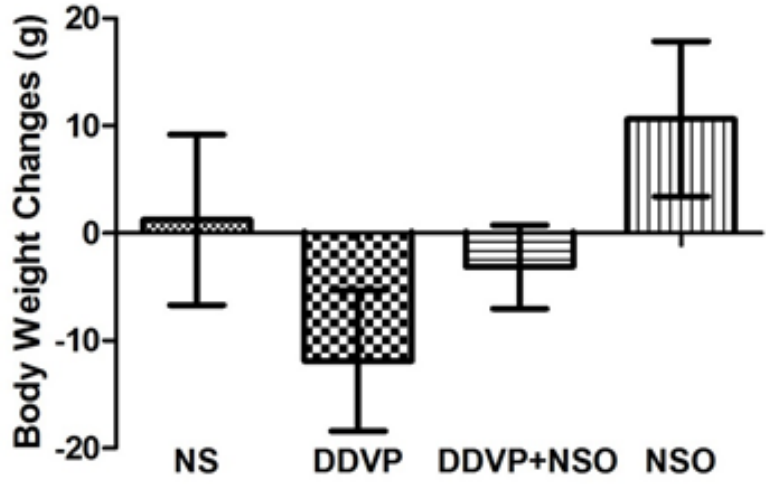

Fig. 1: Change in body weights of rats exposed to Normal saline (NS), Dichlorvos (DDVP), Dichlorvos + Nigella sativa oil (DDVP+NSO) and Nigella sativa oil (NSO).

\subsection{NSO prevented DDVP induced depletion in brain-} body weight ratio

DDVP exposure caused a reduction in brain-body weight ratio, but statistically insignificant from the other groups, however, the co-treatment of DDVP with NSO and NSO alone were able to improve the brain-body weight ratio (Fig. 2).

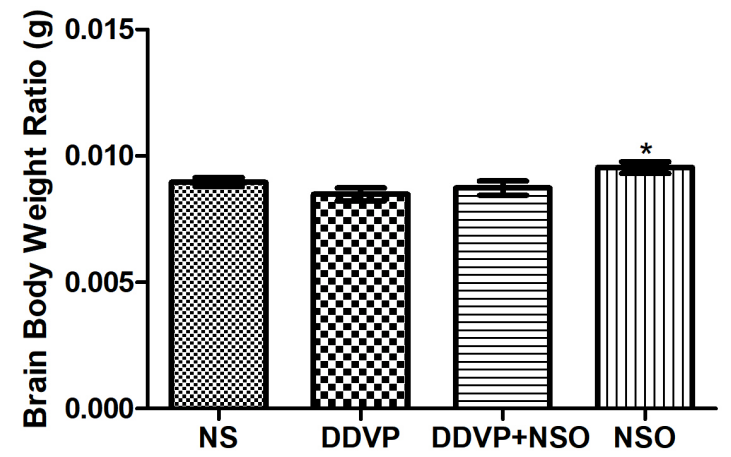

Fig. 2: Average Brain-Body ratio of rats exposed to Normal saline (NS), Dichlorvos (DDVP), Dichlorvos + Nigella sativa oil (DDVP+NSO) and Nigella sativa oil (NSO). Asterisk $(*)$ indicates significant $(p \leq 0.05)$ increase when compared with the DDVP treated rats.

\subsection{NSO reversed DDVP induced NO and ROS outburst in the hippocampus}

Nitric oxide and ROS were significantly elevated in the DDVP only group compared with control. However, significant reduction in NO and ROS levels was observed with NSO intervention in the DDVP+NSO co-exposed group and the NSO only group, when compared with DDVP only (Figs. 3 and 4).

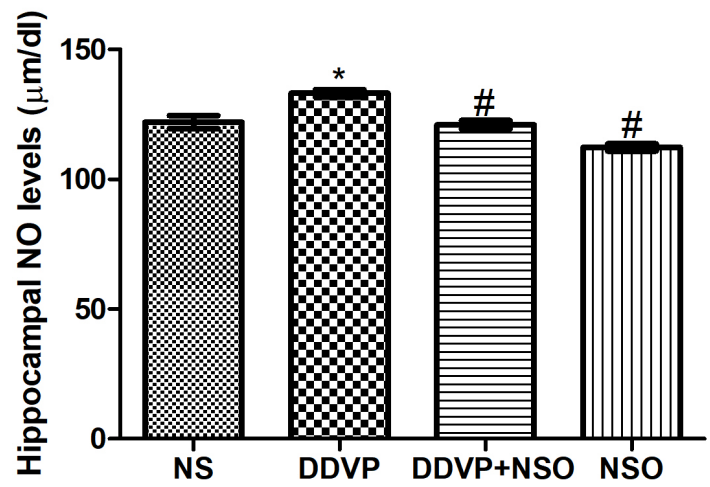

Fig. 3: Hippocampal NO levels in rats exposed to Normal saline, Dichlorvos, Dichlorvos + Nigella sativa oil and Nigella sativa oil. Number sign $(\#)$ indicates significant $(\mathrm{p} \leq 0.05)$ reduction when compared with DDVP exposure, while single asterisk $(*)$ indicates significant $(\mathrm{p} \leq 0.05)$ increase from control and other groups.

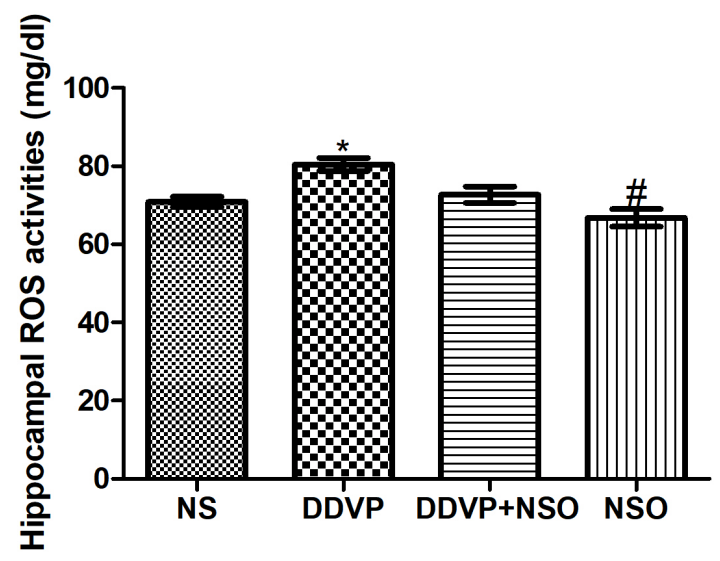

Fig. 4: Hippocampal ROS activities following exposures to Normal saline (NS), Dichlorvos (DDVP), Dichlorvos + Nigella sativa oil (DDVP+NSO) and Nigella sativa oil (NSO). Number sign (\#) indicates significant $(\mathbf{p} \leq \mathbf{0 . 0 5})$ reduction when compared with DDVP exposure, while single asterisk $(*)$ indicates significant $(p \leq 0.05)$ increase from control and other groups.

\subsection{Pro-cholinesterase potentials of NSO against DDVP induced hippocampal AChE deactivation}

Activities of AChE was markedly inhibited in the hippocampus of DDVP exposed rats when compare with the control. Although, AChE activities was also reduced in DDVP+NSO treated rats, it was however not statistically significant when compared with control (Fig. 5). 


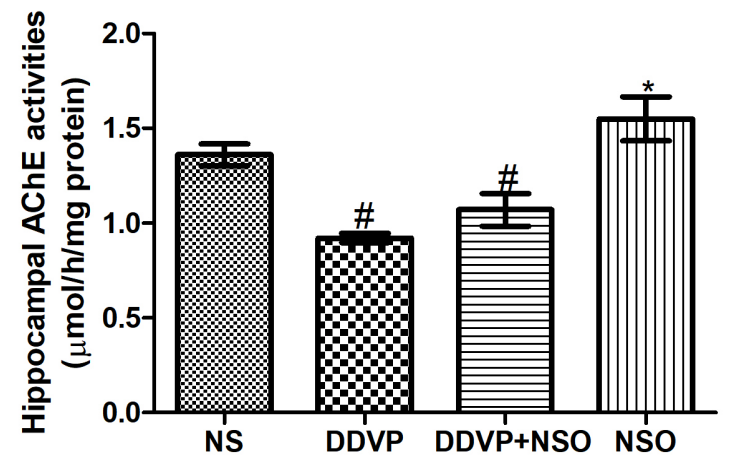

Fig. 5: Hippocampal AChE activities of rats exposed to Normal saline (NS), Dichlorvos (DDVP), Dichlorvos + Nigella sativa oil (DDVP+NSO) and Nigella sativa oil (NSO). Number sign (\#) indicates significant $(p \leq 0.05)$ reduction when compared with both control and NSO treated rats, while single asterisk $(*)$ indicates significant $(p \leq 0.05)$ increase from control and other groups.

\subsection{NSO prevented hippocampal related cognitive decline following DDVP exposures}

A significant increase in escape latency was observed in both the long term and short term memories assessed, following DDVP exposure. The DDVP exposed rats also failed to retrieve the precise location of the hidden platform during the probe trial and spent significantly less time in the target quadrant compared to the control group. Treatment with NSO however, significantly enhanced escape latencies in both LTM and STM trials of the DDVP+ NSO and, NSO only rats. There was also an indication of acquisitions in the rats both NSO only and DDVP+NSO groups as they spent more time in the quadrant of the missing platform during the probe test (Figs. 6, 7 and 8).

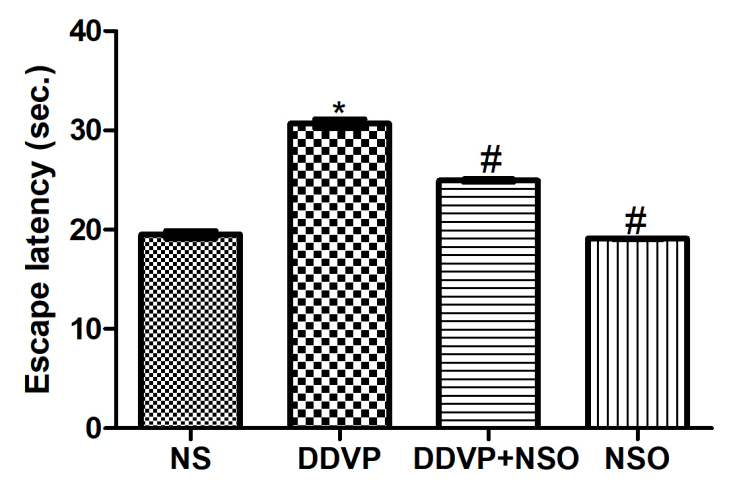

Fig. 6: Escape Latency (LTM) of rats exposed to Normal saline (NS), Dichlorvos (DDVP), Dichlorvos + Nigella sativa oil (DDVP+NSO) and Nigella sativa oil (NSO). Number sign (\#) indicates significant $(p \leq 0.05)$ reduction when compared with DDVP rats, while single asterisk (*) indicates significant $(p \leq 0.05)$ increase from control and the NSO only rats.

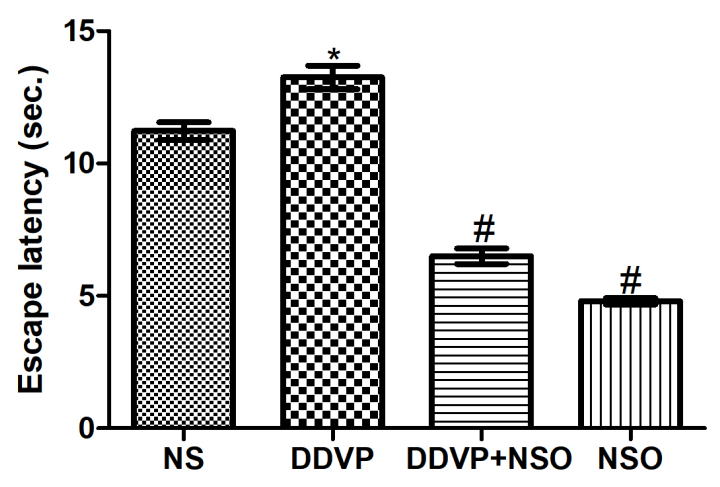

Fig. 7: Escape Latency (STM) of rats exposed to Normal saline (NS), Dichlorvos (DDVP), Dichlorvos + Nigella sativa oil (DDVP+NSO) and Nigella sativa oil (NSO). Number sign (\#) indicates significant $(p \leq 0.05)$ reduction when compared with DDVPand control rats, while single asterisk $(*)$ indicates significant $(p \leq 0.05)$ increase from NSO and DDVP+NSO treated rats.

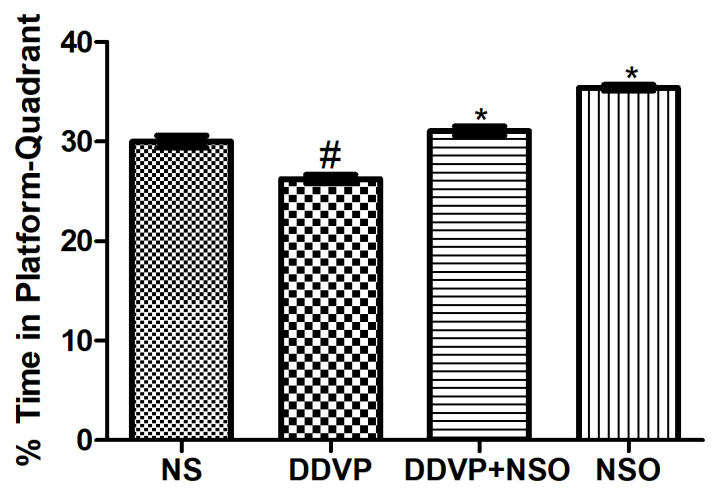

Fig.8: Percentage Time in Quadrant Location (Reference memory) of rats exposed to Normal saline (NS), Dichlorvos (DDVP), Dichlorvos + Nigella sativa oil (DDVP+NSO) and Nigella sativa oil (NSO). Number sign (\#) indicates significant $(p \leq 0.05)$ reduction from all groups, while single asterisk $(*)$ indicates significant $(p \leq 0.05)$ increase from DDVP and or control rats.

\subsection{Protective efficacy of NSO in DDVP induced neurodegenerative like activities}

Exposure to DDVP caused no marked deterioration in the hippocampal subfields (CA 1,2,3 and DG), but few necrotic like vacuolations in the cornu ammonis pyramidal cells in the DDVP only group, these effects were however absent in the DDVP+NSO group (Figs. 9-11). Similarly, a marked loss in density was observed in the neurogenic proteins $(\mathrm{Ki} 67+)$ cells in the hippocampal subfields and the subventricular zone of the DDVP only treated rats, this was also reversed by administration of NSO in the DDVP+NSO rats (Figs. 12-14). 


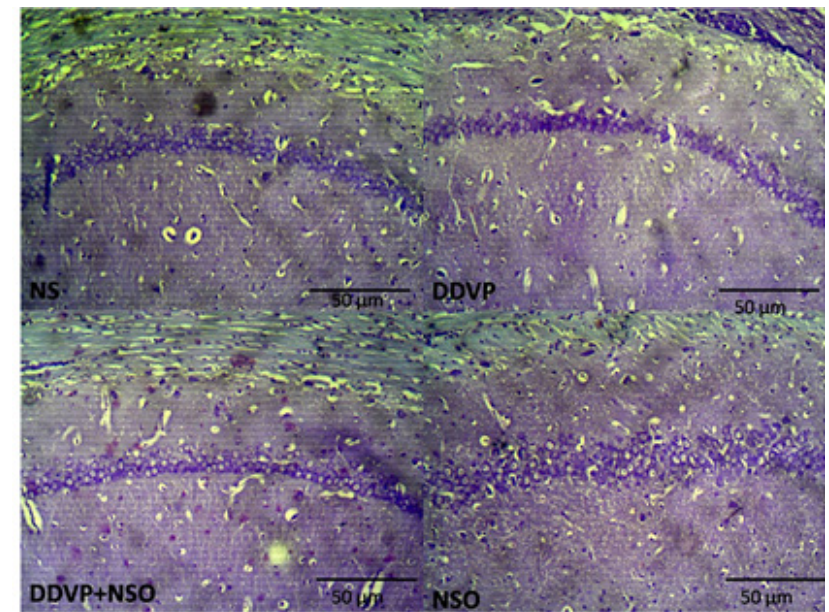

Fig. 9: Representative photomicrographs of cornuammonis 1 sub-field of the hippocampus of rats exposed to Normal saline (NS), Dichlorvos (DDVP), Dichlorvos + Nigella sativa oil (DDVP+NSO) and Nigella sativa oil (NSO). CFV 100X.

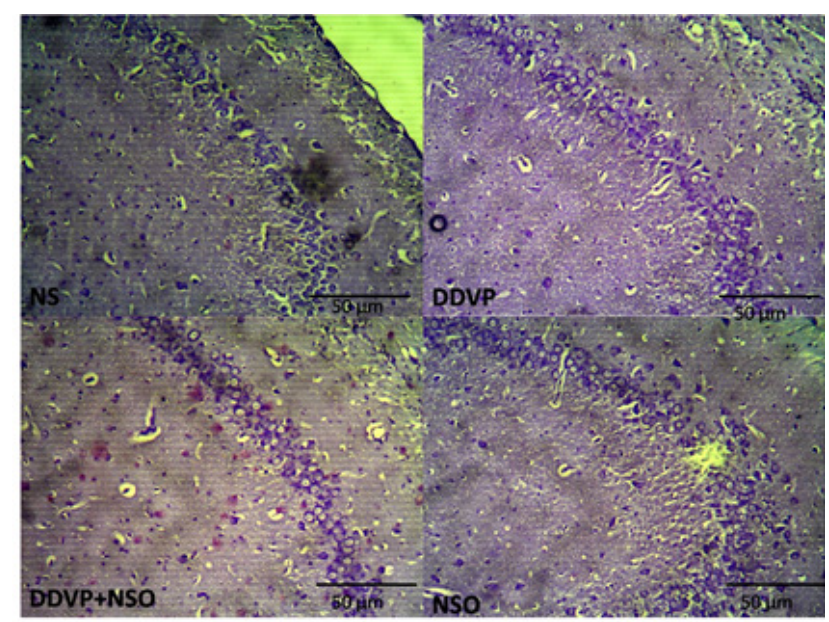

Fig. 10: Representative photomicrographs of cornuammonis 2 sub-field of the hippocampus of rats exposed to Normal saline (NS), Dichlorvos (DDVP), Dichlorvos + Nigella sativa oil (DDVP+NSO) and Nigella sativa oil (NSO). CFV 100X.

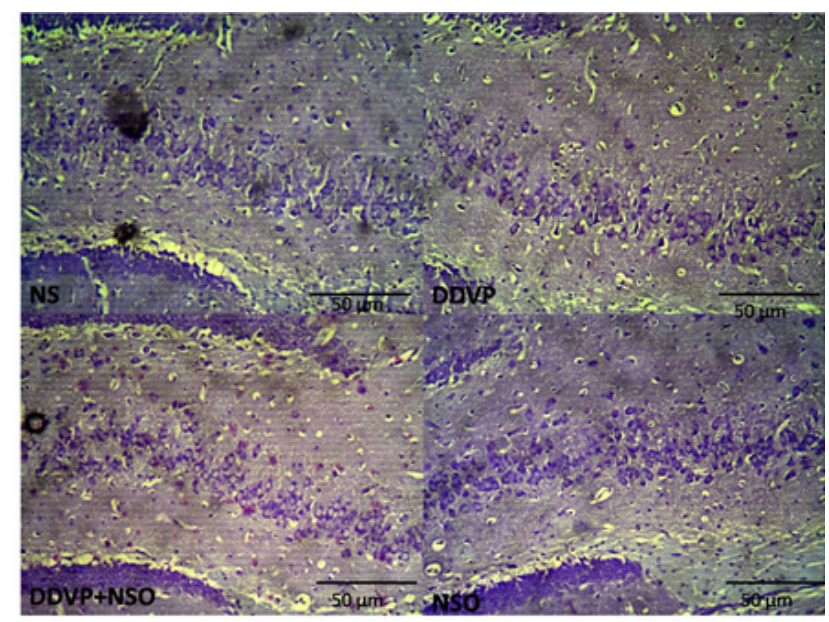

Fig. 11: Representative photomicrographs of cornuammonis 3 sub-field of the hippocampus of rats exposed to Normal saline (NS), Dichlorvos (DDVP), Dichlorvos + Nigella sativa oil (DDVP+NSO) and Nigella sativa oil (NSO). CFV 100X.

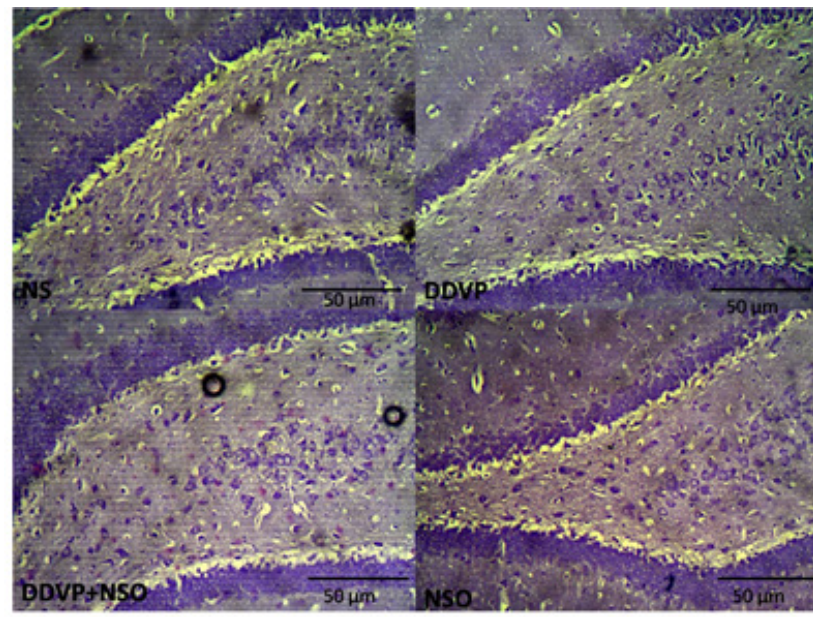

Fig. 12: Representative photomicrographs of dentate gyrus of rats exposed to Normal saline (NS), Dichlorvos (DDVP), Dichlorvos + Nigella sativa oil (DDVP+NSO) and Nigella sativa oil (NSO). CFV 100X.

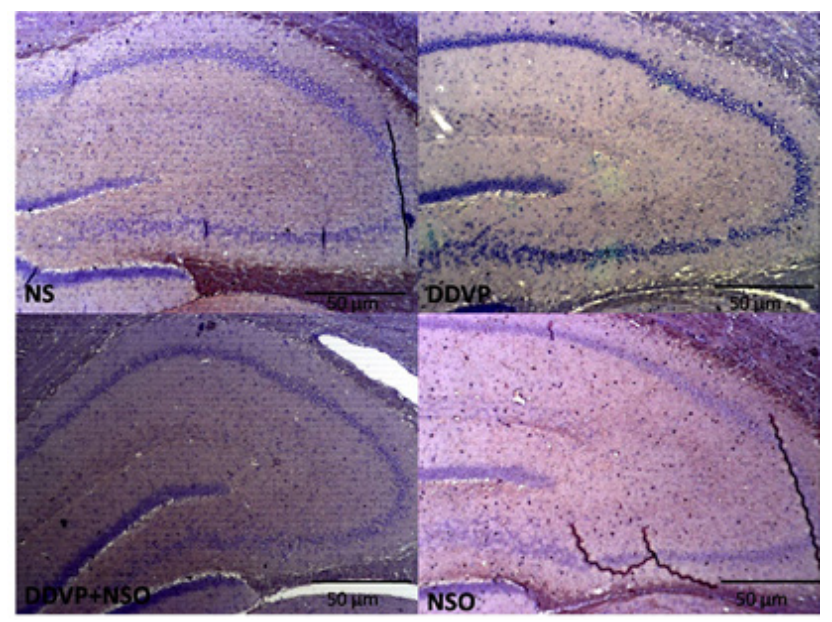

Fig. 13: Representative photomicrographs of the distribution of Ki67 immunoreactive cells in the cornuammonis fields of hippocampus of rats exposed to Normal saline (NS), Dichlorvos (DDVP), Dichlorvos + Nigella sativa oil (DDVP+NSO) and Nigella sativa oil (NSO). Ki67 immunohistochemistry $100 \mathrm{X}$.

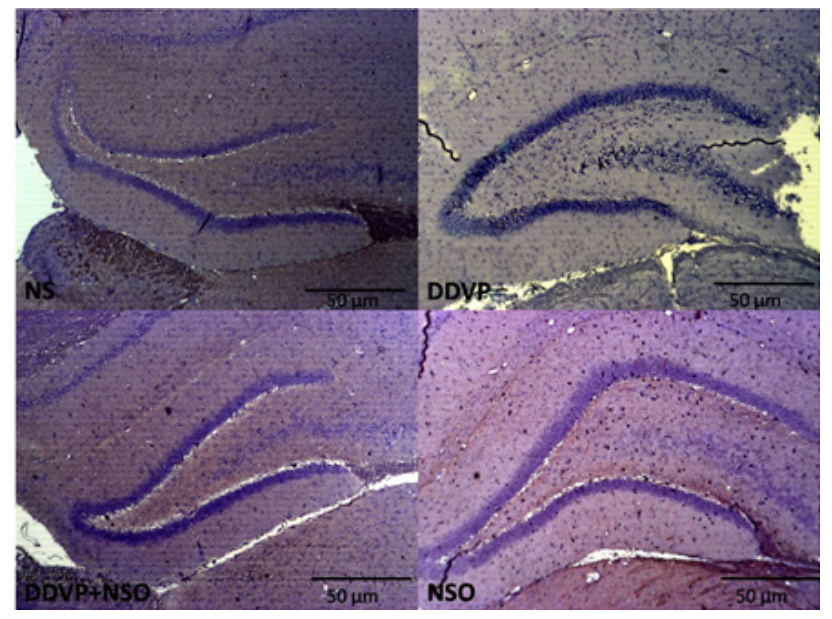

Fig. 14: Representative photomicrographs of the distribution of Ki67 immunoreactive cells in the dentate gyrus of rats exposed to Normal saline (NS), Dichlorvos (DDVP), Dichlorvos + Nigella sativa oil (DDVP+NSO) and Nigella sativa oil (NSO). Ki67 immunohistochemistry 100X. 


\section{DISCUSSION}

There was significant loss of body weight following DDVP exposure, and although no direct changes were observed in the absolute brain weight, but relative brain weight which is a functional marker was markedly reduced with DDVP exposure. These changes were expected considering that previous studies have established a link between pesticide use and loss of brain and body weight. ${ }^{[20]}$

The hippocampal formation consists of temporal lobe circuits that are essential in the survival of an animal being, involved in memory events, spatial and non-spatial cognitive mapping and other functions related to its trisynaptic circuit. ${ }^{[21-23]}$ The hippocampal formation has immense functional importance and has been extensively discovered to contain cellular elements that possess inherent and sustained self-renewal and mitotic capabilities extending into adulthood in the various animal models studied, including humans. ${ }^{[24-26]}$

Cellular lipoidal matrices have been reported as severed or damaged, leading to excessive generation of ROS and subsequent oxidative damages and cell death in OP exposures or poisoning. ${ }^{[12,13,27]}$ In our study, a marked over production of ROS and NO in the hippocampal tissues of the DDVP exposed rats, suggested damage to the cellular membrane of the major cells thus leading to the leak and subsequent oxidative damages in the hippocampus. This finding is consistent with previous reports that submitted that poisoning (inhalation or oral) from OPs exposure caused oxidative damages particularly through the overproduction or ROS in the exposed animals..$^{[12,13,27,28]}$

The extent of the induced oxidative damage was first indicated by the indirect indices (ROS and NO) of oxidative stress recorded in this study. The oxidative damage observed in this study following DDVP exposures caused a relative damage to the cellularity of some of the pyramidal cells in the hippocampal sub-fields, CA3 and CA1, with subsequent neuronal degeneration and vacuolation around these cells. Such effects have been reported following exposure to various OPs which led to pathological changes in different brain regions. ${ }^{[12,13,29]}$

In addition, a marked loss of ki67 positive neurogenic cells in the CA regions, dentate gyrus and the sub-granular zone in the hippocampi of the DDVP exposed rats was observed, and these effects were attributable to oxidative damage. The induced oxidative damage to the hippocampal formation, especially the sub-granular zone and the dentate gyrus could pose deleterious effects on the survival of neurogenic cells (both the neural progenitor cells and the newly generated cells) in these regions. ${ }^{[30]}$

DDVP inhibits AChE activities in the synaptic cleft, causing accumulation of acetylcholine and leading to continuous triggering of the postsynaptic neurons in target and non-target animals is the widely acclaimed mechanism of OPs poisoning. ${ }^{[31]}$ DDVP exposure in the current study impaired $\mathrm{AChE}$ activities in the hippocampus of the exposed rats and in consonant with previous reports, ${ }^{[12,29,32]}$ this would be expected to improve memory function somewhat, considering its pro-cholinergic activities, recognizing the roles of the cholinergic system in the modulation of learning and memory tasks. ${ }^{[33]}$ Nonetheless, the sustained cholinergic stimulation coupled with the accompanying oxidative damage, ${ }^{[12,13,34]}$ does not result in improvements in memory and cognitive performances. This has led to the propositions that the chronic effects of OPs exposures likely occur through mechanisms other than AChE inhibition in the absence of marked cholinesterase severities. ${ }^{[35]}$ And confirming this proposition in the present study, there was evidence of disruption in the spatial memory indices following DDVP exposure. DDVP caused a marked delay in the time the exposed rat took to find the hidden platform, both in the long term and short term memory trials, after the acquisition trials. There was also a great loss in the referential memory index of the rats as they were unable to locate and explore the quadrant that previously contained the platform.

The observed damages in memory indices could further be associated with the extent of loss in the neurogenic cells in the hippocampus, which contain the crucial circuits for memory or cognitive functions, more so, that there is an established link between dysregulated neurogenesis and neurocognitive diseases. ${ }^{[36]}$ Furthermore, DDVP and other OPs poisoning have been reported to sever personality, impair neurocognitive tasks and cause psychocognitive derangements, ${ }^{[12,37]}$ coupled with neurogenic cells loss in related brain region. ${ }^{[12]}$

Nigella sativa oil (NSO) being a natural antioxidant essential oil, ${ }^{[12,13]}$ with proven neuro-protective efficacies, ${ }^{[12,13,15,18.38]}$ was explored as a potent neuroprotective substance against DDVP neurotoxicity. And effectively, NSO intervention in this study was vital in preventing the out-burst of both ROS and NO productions in the hippocampus of the DDVP and/ or NSO exposed rats, an effect that could be supported by its previously reported antioxidant efficacy against models of neurotoxicity ${ }^{[12,13,19,39-41]}$ The antioxidant effects of NSO against DDVP we observed in this study as well as those reported in other neurotoxic models, ${ }^{[12,13]}$ further strengthen the efficacy of NSO against OPinduced neurological deficits. ${ }^{[42,43]}$ Co-administration of NSO with DDVP also revealed that NSO was able to preserve the cholinergic integrity of the hippocampus, by minimizing AChE deactivation induced by DDVP in the exposed rats. Although, NSO improved AChE activities in this work, there are reports that NSO and/ or its constituent thymoquinone possess moderate $\mathrm{AChE}$ inhibitions activities, implicated in memory function tasks, ${ }^{[44]}$ a cholinergic interaction that may be linked to its efficacy against neuro-cognitive, neuropsychiatry and neurodegenerative impairments ${ }^{[12,15,18,38]}$

Potency of the AChE reactivation and antioxidant capacities of NSO observed in this study against DDVP neurotoxicity were further evident in the improved relative 
brain weight and memory indices in the NSO treated rats, either with or without DDVP exposure, a report similar to what is available in the literature about the efficacy of Nigella sativa in enhancing cognitive functions. [12,15,18,38,45,46] Complementing its neurocognitive activities, NSO treatment preserved the histological integrities of hippocampal subfield CA regions pyramidal cells and dentate gyrus granule cells and such functions are evident in its beneficial efficacy against degenerative exposures to neurotoxic models. ${ }^{[12,13,15,18,38,47-49]}$ NSO also enhanced the survival of neurogenic cells in the hippocampus, which may have contributed to improved behavioural performances by the rats in memory related activities. NSO and/or its constituent thymoquinone are extensively reported to restore neuronal integrity and functions by increasing neuronal density, ${ }^{[47]}$ decreasing apoptosis and preventing inflammatory processes. ${ }^{[4]]}$

\section{CONCLUSION}

All of the above effects of the Nigella sativa oil can be associated with its antioxidant capacities which prevent oxidative damages, a process implicated in the initiation of pathological processes in various neurological diseases. ${ }^{[19,39-42]}$ Therefore we conclude that interventional administration of NSO in this study had hippocampal neuroprotective functions against the effects of DDVP exposure, and that this predominantly occurred through the modulation of oxidative and neurogenic functions.

\section{ACKNOWLEDGEMENT}

The authors appreciate the research incentives from the Deanship Research support of the Faculty of Basic Medical Sciences, University of Ilorin, Ilorin, Nigeria. Also, the technical assistance of Mrs Hasiena Ali of the immunohistochemistry and histology laboratory, School of Anatomical Sciences, Faculty of Health Sciences, University of the Witwatersrand, South Africa.

\section{CONFLICT OF INTEREST}

There are no conflicts of interest.

\section{REFERENCES}

1. C.P. Holstege, A.B. Baer, Insecticides, Curr. Treat. Options. Neurol. 6 (2004) 17-23.

2. K.M. Hayden, M.C. Norton, D. Darcey, T. Ostbye, P.P. Zandi, J.C. Breitner, K.A. Welsh-Bohmer, Occupational exposure to pesticides increases the risk of incident AD: The Cache County study, Neurology. 74 (2010) 1524-1530.

3. T.A. Slotkin, Does early-life exposure to organophosphate insecticides lead to prediabetes and obesity?, ReprodToxicol. 31 (2011) 297-301.

4. T. Fariba, V. Gholamhassan, A. Mohammad, A.M. Ali, A Comparative Study of the Quality of Life, Depression, Anxiety and Stress in Farmers
Exposed to Organophosphate Pesticides with those in a Control Group, J. Chem. Health. Risks. 6(2) (2016) 143-151

5. Y. Oulhote, M.F. Bouchard, Urinary metabolites of organophosphate and pyrethroid pesticides and behavioral problems in Canadian children, Environ. Health. Perspect. 121 (2013) 1378-1384.

6. Y. Kalender, S. Kaya, D. Durak, F.G. Uzun, F. Demir, Protective effects of catechin and quercetin on antioxidant status, lipid peroxidation and testis histoarchitecture induced by Dichlorvos in male rats, Environ. Toxicol. Pathol. 33 (2012) 141-148.

7. M.S. Ajao, S. Adebayo, A. Imam, A. Ibrahim, M.Y. Adana, A. Alli-Oluwafuyi, S.B. Kareem, Protective Effect of Nigella Sativa (Black Caraway) Oil on Oral Dichlorrvos Induced Hematological, Renal and Nonspecific Immune System Toxicity in Wistar rats, Iran. J. Toxic. 11(6) (2017) 1-5.

8. H. Bas, Y. Kalender, Dichlorvos induced cardiotoxicity in rats and the protective role of quercetin and catechin, GUJS. 24(3) (2011) 387-395.

9. J.Y. Khokhar, R.F. Tyndale, Rat brain CYP2Benzymatic activation of Dichlorvos to the oxon mediates cholinergic neurotoxicity, Toxicol. Sci. 126(2) (2012) 325-335.

10. W.J. Hendelman, Atlas of Functional Neuroanatomy, CRC Press LLC: BocaRaton, 2000, pp. 187-199.

11. C. Vivar, H. VanPraag, Functional circuits of new neurons in the dentate gyrus, Front. Neural. Circuits. 7 (2013) 15 doi: 10.3389/fncir.2013.00015.

12. A. Imam, N.A. Sulaiman, A.L. Oyewole, A. Amin, S-T.T. Shittu, M.S. Ajao, Pro-Neurogenic and Antioxidant Efficacy of Nigella sativa Oil reduced Vulnerability to Cholinesterase Dysfunction and Disruption in Amygdala Dependent Behaviors in CPF Exposure, JKIMSU. 7(3) (2018a) 1-12.

13. A. Imam, A. Ogunniyi, A. Ibrahim, W.I. Abdulmajeed, L.A. Oyewole, A.H. Lawan, F.A. Sulaimon, M.Y. Adana, M.S. Ajao, Dichlorvos induced Oxidative and Neuronal responses in rats: Mitigative Efficacy of Nigella sativa (black cumin), Niger. J. Physiol. Sci. 33 (2018b) 83-88.

14. M. Alemi, F. Sabouni, F. Sanjarian, K. Haghbeen, S. Ansari, Anti-inflammatory effect of seeds and callus of Nigella sativa L. extracts on mixglial cells with regard to their thymoquinone content, AAPS. PharmSciTech. 14 (2013) 160-167.

15. M.S. Ajao, A. Imam, A. Amin, W.I. Abdulmajeed, 
M.I. Ajibola, A. Alli-oluwafuyi, W.G. Balogun, O.J. Olajide, A. Ibrahim, Black Seed Oil Improves Motor and anxiety like Behaviours and Cerebellar Cyto-Architectonic in Male Wistar Rats, Nig. J. Neurosci. 8(1) (2016a) 8-14.

16. E.I. Salim, Cancer chemo preventive potential of volatile oil from black cumin seeds, Nigella sativa L., in a rat multi-organ carcinogenesis bioassay, Oncol. Let. 1 (2010) 913-924.

17. A.H.Alhebshi, M. Gotoh, I. Suzuki, Thymoquinone protects cultured rat primary neurons against amyloid $\beta$-induced neurotoxicity. Biochem. Biophys. Res. Commun. 19 (2013) 362-367.

18. A. Imam, M.S. Ajao, M.I. Ajibola, A. Amin, W.I. Abdulmajeed, A.Z. Lawal, A. Alli-Oluwafuyi, O.B. Akinola, A.O. Oyewopo, O.J. Olajide, M.Y. Adana, Black seed oil ameliorated scopolamineinduced memory dysfunction and corticohippocampal neural alterations in male Wistar rats. Bulletin of Faculty of Pharmacy, Cairo University (2016b) 54, 49-57.

19. R. Bargi, F. Asgharzadeh, F. Beheshti, M. Hosseini, H.R. Sadeghnia, M. Khazaei M, The effects of thymoquinone on hippocampal cytokine level, brain oxidative stress status and memory deficits induced by lipopolysaccharide in rats, Cytokine. 96 (2017) 173-184.

20. G.L. Jr. Kennedy, Chronic toxicity, reproductive, and teratogenic studies with oxamyl, Fundam. Appl. Toxicol. 7(1) (1986) 106-118.

21. V. Manuel, M-d-1-P. Liset, The hippocampus in depth: a sublayer-specific perspective of entorhinal-hippocampal function, Curr. Opin. Neurobiol. 52 (2018) 107-114.

22. C.M. Bird, The role of the hippocampus in recognition memory, Cortex. 93 (2017) 155-165.

23. H. Eichenbaum, The role of the hippocampus in navigation is memory, J. Neurophysiol. 117 (2017) 1785-1796.

24. P. Gregor-Alexander, B. Sara, B. Marion, J.J. David, C. Stefano, D.S. Benjamin, H. Fritjof, J. Sebastian, Live imaging of neurogenesis in the adult mouse hippocampus, Science. 359 (2018) 658-662.

25. C. Anacker, R. Hen, Adult hippocampal neurogenesis and cognitive flexibility - linking memory and mood, Nat. Rev. Neurosci. 18 (2017) 335-346.

26. C. Jorgensen, Adult Mammalian Neurogenesis and Motivated Behaviors, Integr. Zool. (2018); doi: 10.1111/1749-4877.12335

27. P. Sharma, R. Singh, Dichlorvos and lindane induced oxidative stress in rat brain: protective effects of ginger, Pharmacog. Res. 4(1) (2012) 27 32 .

28. N. Aly, K. El-Gendy, F. Mahmoud, A.K. El-Sebae, Protective effect of vitamin $\mathrm{C}$ against Dichlorvos oxidative stress in male mice, Pestic. Biochem. Phys. 97 (2010) 7-12.

29. M.E. Omar, E.R. Abdel-Salam, R.S.E. Youness, N.A. Esmail, Y.A. Mohammed, A. Khadrawy, A. Sleem, M.A. Amany, Methylene Blue as a Novel Neuroprotectant in Acute Malathion Intoxication, React. Oxy. Spec. 1(2) (2016) 165-177.

30. V.C. Piatti, L.A. Ewell, J.K. Leutgeb, Neurogenesis in the dentate gyrus: carrying the message or dictating the tone, Front. Neurosci. 7 (2013) 50. doi: 10.3389/fnins.2013.00050

31. S. Das, A review of dichlorvos toxicity in fish, Curr. World. Environ. 8(1) (2013) 143-149.

32. C. Diana, L. Caridad, C. Fernando, L. Jordi, F. Pilar, P. Floria, S. Fernando, Dose-dependent regional brain acetylcholinesterase and acylpeptide hydrolase inhibition without cell death after chlorpyrifos administration, J. toxicol. Sci. 38(2) (2013) 193-203.

33. J. Araujo, N. Greig, Cholinesterase inhibitors improve both memory and complex learning in aged beagle dogs, J. Alzheimers. Dis. 26(1) (2010) 143-55.

34. L. Asma, K. Mohamed, L. Zohra, R. Rachid, F. Hamadi, C. Yassine, D. Zama, S. Rachid, Neurobehavioral deficits and brain oxidative stress induced by chronic low dose exposure of Persistent Organic Pollutants mixture in adult female rat, Environ. SciPollut. Res. (2016) doi:10.1007/ s11356-016-6913-9

35. M. Iris, V. Eugenio, E. Jorge, C.C.F. Tanos, Neurotoxic Effects Associated with Current Uses of Organophosphorus Compounds, J. BrazChemSoc. 27(5) (2016) 809-825.

36. S. Yun, R.P. Reynolds, I. Masiulis, A.J. Eisch, Re-evaluating the link between neuropsychiatric disorders and dysregulated adult neurogenesis, Nat. Med. 22 (2016) 1239-1247.

37. A.S. Alessandra, A.N. Aline, de O. Jade, C. Dirleise, B.S. Danúbia, A.H. Mariana, L.G.M. Eduardo, S. Cristina, F.B. Andreza, F. Marcelo, Long-term and low-dose malathion exposure causes cognitive impairment in adult mice: evidence of hippocampal mitochondrial dysfunction, astrogliosis and apoptotic events, Arch. Toxicol. 90 (2016) 647. doi:10.1007/s00204-015-1466-0

38. A. Imam, M.S. Ajao, A. Amin, W.I. Abdulmajeed, 
M.I. Ajibola, A. Ibrahim, O.J. Olajide, W.G. Balogun, Cannabis Induced Moto-Cognitive Dysfunctions in Wistar Rats: Ameliorative efficacy of Nigella sativa, Malays. J. Med. Sci. 23(5) (2016c) 17-28.

39. A. Abbasnezhad, P. Hayatdavoudi, S. Niazmand, M. Mahmoudabady, The effects of hydroalcoholic extract of Nigella sativa seed on oxidative stress in hippocampus of STZ-induced diabetic rats, Avicenna. J. Phytomed. 5 (2015) 333-340.

40. M.M. Safhi, Neuromodulatory effects of thymoquinone in extenuating oxidative stress in chlorpromazine treated rats, Acta. Pol. Pharm. 73 (2016) 529-535.

41. Y.Y. Shao YY, B. Li, Y.M. Huang, Q. Luo, Y.M. Xie, Y.H. Chen, Thymoquinone attenuates brain injury via an anti-oxidative pathway in a status epilepticus rat model, Transl. Neurosci. 8 (2017) 9-14.

42. E.A. Manal,A.A. Elhalwagy, R.M.Z. Nahas, E.A.F. Hoda, Potential Effect of Vitamin $C$ and Curcumin on Oxidative Stress and Skin Lesion Induced by Dermal Intoxication with Cypermethrin, AmerChemSci. J. 8(1) (2015) 1-12.

43. M.N. Syed, O. Shreesh, T. Kornelia, S. Mohammad, K. Huba, A. Abdu, Efficacy of N-Acetylcysteine, Glutathione, and Ascorbic Acid in Acute Toxicity of Paraoxon to Wistar Rats: Survival Study, Oxi. Med. Cell. Long. (2015); http://dx.doi.org/10.1155/2015/329306

44. M. Hosseini, pour T. Mohammad, R. Karami, Z. Rajaei, H.R. Sadeghnia et al., Effects of the hydro-alcoholic extract of Nigella Sativa on scopolamine-induced spatial memory impairment in rats and its possible mechanism, Chin. J. Integ. Med. 21 (2015) 438-444.

45. M.K. Sahak, A.M. Mohamed, N.H. Hashim, Adli. D.S. Hasan, Nigella Sativa oil enhances the spatial working memory performance of rats on a radial arm maze, Evid. Based. Complement. Alternat. Med. 5 (2013) doi:10.1155/2013/180598

46. F. Beheshti, M. Hosseini, F. Vafaee, M.N. Shafei, M. Soukhtanloo, Feeding of Nigella Sativa during neonatal and juvenile growth improves learning and memory of rats, J. TradCompl. Med. (2015) 725-733.

47. I. Gulsen, H. Ak, N. Colcimen, H.H. Alp, M.E. Akyol, İ. Demir, T. Atalay, R. Balahroğlu, M.C. Rağbetli, Neuroprotective effects of thymoquinone on the hippocampus in a rat model of traumatic brain injury, World. Neurosurg. 86 (2016) 243-249.

48. M.F. Heba, A.N. Neveen, F.M. Faten, A.E. Anwar, M.R. Nasr, Nigella sativa as an anti-inflammatory and promising remyelinating agent in the cortex and hippocampus of experimental autoimmune encephalomyelitis-induced rats, Int. J. Clinic. Exp. Path. 2015;8(6):6269-6286

49. I. Norsharina, I. Maznah, A.L. Latiffah, M. Musalmah, A.M. Abdalbasit, Black Cumin Seed (Nigella Sativa Linn.) Oil and its Fractions Protect against Beta Amyloid Peptide-Induced Toxicity in Primary Cerebellar Granule Neurons, J. Food. Lipids. 15(4) (2008). DOI: $10.1111 / \mathrm{j} .1745-4522.2008 .00137$ 